Revista de la Escuela de Ciencias de la Educación, año 13, nRo. 12, vol. 2, julio a diciembre de 2017. Páginas 129147. ISSN 1851-6297. ISSN 2362-3349 (EN LínEA). DEMOCRATIZAR EL GOBIERNo DE LA EDUCACIÓN. HISTORIA RECIENTE Y coyunturas criticas del subsistema educativo en Santa Fe. Mariano Sironi.

\title{
DEMOCRATIZAR EL GOBIERNO DE LA EDUCACIÓN. HISTORIA RECIENTE Y COYUNTURAS CRÍTICAS DEL SUBSISTEMA EDUCATIVO EN SANTA FE
}

\author{
Mariano Sironi* \\ Universidad Nacional de Rosario, Argentina. \\ marianosironi@yahoo.com.ar
}

Recibido: 20/09/2016 Aceptado: 22/02/2016

\section{Resumen}

El trabajo presenta reflexiones sobre el Gobierno del sistema educativo santafesino inscribiéndose en la agenda abierta dentro del campo de las ciencias sociales a partir de los procesos de descentralización administrativa que abrieron el juego a análisis y reconstrucciones de procesos políticos e históricos en otra escala, la subnacional.

El objetivo de este trabajo es describir coyunturas críticas en las que se constituyó el subsistema de gobierno de la educación y la dependencia del rumbo (path dependence) del sistema educativo de la provincia de Santa $\mathrm{Fe}$ en el marco de un nuevo debate sobre la sanción de una ley provincial de educación.

Se propone una reconstrucción de coyunturas críticas que configuraron regularidades y fortalecieron actores en la institucionalidad del gobierno de la educación de carácter centralizada que se consolida durante las dictaduras militares de 1966-1973 y 1976-1983, que persisten en el proceso abierto a partir de 1983. El trabajo hace foco en particular en el Consejo General de Educación, organismo creado en 1886 y desmantelado de manera definitiva

Profesor de grado Superior en Ciencia Política. Facultad de Ciencia Política y Relaciones Internacionales. UNR. Licenciado en Ciencia Política. Facultad de Ciencia Política y Relaciones Internacionales. UNR. Doctorando en Humanidades y Artes con Mención en Educación - Facultad de Humanidades y Artes, UNR. (En proceso de escritura de Tesis). Titular interino de la cátedra de Historia de la Educación del Profesorado en Psicología. Facultad de Psicología. UNR. 
durante la última dictadura militar, cuyo restablecimiento se encuentra propuesto en diversos proyectos de Ley Provincial de Educación e implica la posibilidad de ampliar los actores participantes en los procesos de toma de decisiones.

Palabras Clave: Gobierno de la Educación - Consejo Provincial de Educación - Coyunturas críticas - Santa Fe (Argentina).

\section{Abstract}

The paper presents reflections on the Government of Santa Fe educational system by registering on the agenda open within the field of social sciences from the processes of administrative decentralization that opened the game analysis and reconstructions of political and historical in another scale processes, sub. The aim of this paper is to describe critical junctures where the subsystem government education and dependence on path (path dependence) of the educational system of the province of Santa Fe in the framework of a new debate on the sanction was established a provincial education law. a reconstruction of regularities critical junctures that shaped and strengthened the institutional actors in government education centralized character that is consolidated during the military dictatorships of 1966-1973 and 1976-1983 that persist in the open process from 1983 is proposed. the work does focus in particular on the General Education Council, a body created in 1886 and dismantled definitively during the last military dictatorship, whose reinstatement is proposed in various projects Provincial Education Act and involves the possibility of extending the actors participants in the decision-making processes.

Keywords: Government Education - Provincial Board of Education - Critical junctures - Santa Fe (Argentina).

\section{Introducción}

El trabajo presenta reflexiones sobre la configuración del gobierno de la educación en el sistema educativo de la provincia de Santa Fe, uno de los más grandes de la Argentina (1).

Proponemos describir el proceso de desmantelamiento del Consejo General de Educación, organismo colegiado creado en el marco de la Ley de Educación Común de la Provincia (1886) que funcionó de manera discontinua hasta el advenimiento del Terrorismo de Estado (1976-1983). Proponemos como hipótesis que dicho desmantelamiento se produjo en dos coyunturas críticas claves como fueron las dictaduras militares argentinas de 1966-1973 y 1976-1983, dando lugar a un proceso de centralización en la toma de decisiones en esta área del estado donde, además, el sector de la educación privada ganará en autonomía a partir de una ley dictada en 1968, la Ley Nº 6247 que 
Revista de la Escuela de Ciencias de la Educación, año 13, nRo. 12, vol. 2, julio a diciembre de 2017. Páginas 129147. ISSN 1851-6297. ISSN 2362-3349 (EN LínEA). DEMOCRATIZAR EL GOBIERNo dE LA EDUCACIÓN. HISTORIA RECIENTE Y coyunturas criticas del subsistema educativo en Santa Fe. Mariano Sironi.

crea el Servicio Provincial de Educación Privada. Dicha institucionalidad será ratificada, como veremos, por normas de gobiernos justicialistas electos a partir de 1983 y tendrá como principal beneficiario a la Iglesia Católica.

Para poder comprender qué cuestiones cambian en estas coyunturas críticas debemos referirnos a normas y políticas educativas anteriores como fueron las reformas educativas de los gobiernos Justicialistas y los que lo sucedieron luego del derrocamiento de Perón en setiembre de 1955. Fundamentalmente los gobiernos llamados desarrollistas de Silvestre Begnis (19581962) y de Tessio (1963-1966).

El trabajo extiende la mirada hasta nuestro presente por los motivos que planteamos a continuación.

Nuestras reflexiones se insertan en dos agendas, en primer lugar, en la Agenda pública actual, teniendo en cuenta que la provincia de Santa Fe se encuentra deliberando acerca de la necesidad de una nueva ley Provincial de Educación (2) inaugurando un proceso político inédito en la provincia desde 1983 en adelante ya que Santa Fe y la Ciudad Autónoma de Buenos Aires son las únicas Jurisdicciones subnacionales que no han sancionado leyes provinciales de educación para su respectivo territorio. Aunque vale aclarar que en el caso de nuestra provincia ha habido adhesiones de la legislatura provincial a las Leyes educativas nacionales sancionadas por el Congreso de la Nación en 1995 (Ley N²4.195) y 2006 (Ley N²6.206).

A su vez, de los cuatro proyectos de ley presentados en la legislatura provincial, tres se proponen restablecer un Consejo Provincial o General de Educación dependiendo del proyecto que se elija, lo cual nos habla de la existencia de sectores que buscan reivindicar esta instancia decisoria para el gobierno de la educación. De este modo, este artículo se propone como un aporte a las deliberaciones públicas en curso alrededor de esta agenda pública.

En segundo lugar, pretendemos realizar un aporte a la agenda de investigación centrada en los niveles subnacionales del Estado que intenta clarificar las transformaciones de las últimas décadas a partir de las reformas estatales uno de cuyos pilares han sido los procesos de descentralización administrativa.

En este sentido, nos interesa aportar al fortalecimiento del diálogo entre una corriente de la Ciencia Política (el institucionalismo histórico) y la Política Educacional como ya lo vienen realizando clásicos autores de este campo postulando la necesidad de estudiar la gobernabilidad de los sistemas desde enfoques jurídico normativos (Paviglianiti, 1996, Ruiz, 2013) centrando la mirada en un nivel del Estado, el provincial.

Lardone y González al referirse a la importancia de investigar dicho nivel plantean que

(...) uno de los motivos centrales de este desarrollo, (...) es la intuición de que conocer y estudiar la política subnacional es fundamental para com- 
prender la política argentina en sentido amplio. Más aún, es la constatación de que los procesos políticos nacionales no pueden ser comprendidos y explicados si no atendemos a la dimensión subnacional de dichos procesos. En ella está una parte importante de los factores que explican los cambios experimentados en los últimos años en diversas áreas. La dimensión subnacional es importante para comprender el rediseño de las relaciones intergubernamentales, para explicar variaciones en la calidad de las políticas públicas (Lardone y Gonzalez, 2013, p. 333).

Es sabido que en nuestro país se produjeron transferencias de establecimientos educativos de los niveles primario, secundario y superior no universitario pertenecientes a la jurisdicción nacional en dos fases. La primera se da en los años 1978-1980 durante el Proceso de Reorganización Nacional y la segunda ola de transferencias se produjo a partir de la sanción de la Ley $\mathrm{N}^{\circ}$ 24.049 del año 1991.

La literatura especializada caracteriza estos procesos de transferencia como descentralización educativa, la cual estuvo motivada y orientada por razones y lógicas de índole economicista (Filmus 1997, Falleti, 2004). Asimismo, las transferencias implicaron un fenómeno en el que coexisten mecanismos centralizados de imponer las políticas desde el nivel nacional con nuevas formas de regulación del sistema (por ejemplo, la Concertación). Es decir, el Estado Nacional legitima las decisiones tomadas en el nivel central en el ámbito del Consejo Federal de Educación y desde el mismo, coordina y evalúa las políticas, mientras que las provincias se responsabilizan de la implementación de las mismas (Pronko-Vior, 1999; Plomé, 2002; Nosiglia-Rebello, 2005; Gorostiaga, 2007; Rivas, 2004; Gorostiaga y Vieyra, 2011).

Otros trabajos plantean que paralelamente a la asunción de este nuevo rol del Estado Nacional se produjo una recentralización al interior de las provincias (Feldfeber, 2009; Carletti, 2005). Sin embargo, y sin diferir con ese planteo crítico, entendemos que en el caso específico de Santa Fe la recentralización como forma de gobierno del sistema educativo es un proceso previo que se acopla y refuerza con lo acontecido en los '90, conjuntamente con la consolidación de cierta autonomía del sector privado.

En el presente trabajo partimos de la idea de que la mencionada institucionalidad, se construyó durante las coyunturas críticas mencionadas. Esta conceptualización nos permitirá señalar el proceso de construcción de un rumbo institucional cuyas persistencias y consolidaciones se dieron en democracia en términos normativos.

En este sentido, el desafío que se presenta para quienes quieran reformar en sentido democrático la educación en Santa Fe es mayúsculo porque implicará discutir acerca de distribuir (o no) poder de decisión acerca de las cuestiones educativas en una provincia que, como veremos, tiene una institu- 
Revista de la Escuela de Ciencias de la Educación, año 13, nRo. 12, vol. 2, julio a diciembre de 2017. Páginas 129147. ISSN 1851-6297. ISSN 2362-3349 (EN LínEA). DEMOCRATIZAR EL GOBIERNo DE LA EDUCACIÓN. HISTORIA RECIENTE Y coyunturas criticas del subsistema educativo en Santa Fe. Mariano Sironi.

cionalidad donde el gobierno de la educación es centralizado.

Para ello dividimos el trabajo en cinco apartados en el primero realizamos algunas aclaraciones conceptuales. En los apartados siguientes presentamos las sucesivas coyunturas críticas para finalizar con algunas conclusiones provisorias.

\section{Algunas referencias conceptuales acerca del gobierno de la Educación}

En este apartado nos interesa dar cuenta de las referencias conceptuales presentes en el recorrido que proponemos.

El Gobierno de la educación ya sea a nivel de lo nacional o de lo subnacional abarca una amplia bibliografía de donde podemos destacar los trabajos de Rivas (2004), Grinberg (2008), Gvirtz (2008), Feldfeber (2009), Abratte (2010) y Giovine (2012) entre otros. En los mismos no hay una conceptualización del Gobierno de la Educación compartida, y se destaca el diálogo con otras disciplinas. En nuestro caso, entendemos al Gobierno de la educación como un subsistema del Sistema Educativo compuesto por un conjunto de instituciones y actores que estructuran la toma de decisiones obligatorias para las organizaciones e individuos que componen el sistema de manera durable y regular. Consecuentemente este subsistema es entendido como el mecanismo o estructura de producción de políticas educativas (Acuña y Chudnovsky, 2007), en el cual intervienen instituciones formales - forma de gobierno - e instituciones informales - modo de gobernar - (O'Donnell, 2010) que regulan el proceso de toma de decisiones.

De este modo, al analizar un subsistema de gobierno de la educación estamos indagando acerca de la manera y el grado en que se pueden promover los intereses y los valores particulares de determinados actores implicados en el proceso educativo. De allí que el debate sobre una Ley Provincial de Educación y por ende el diseño institucional del sistema educativo adquiere connotaciones de controversia, debido a las importantes consecuencias distributivas que conlleva.

Es decir, en definitiva la cuestión nos vincula a la pregunta sobre la interacción entre los actores sociopolíticos y las instituciones.

Dentro de la Ciencia Política en las últimas décadas se produjo una revalorización de la importancia de las instituciones para entender los procesos políticos. Como señala Peters (2003) las instituciones son un rasgo estructural de las sociedades o de las formas de gobierno de las mismas, su estabilidad y duración en el tiempo es un atributo ineludible y, fundamentalmente, afectan y/o condicionan los comportamientos individuales y/o colectivos.

En este marco, el neo-institucionalismo histórico (3) se preocupa por estudiar el origen, la evolución y las consecuencias de las instituciones políticas en general y como estas condicionan y/o determinan las decisiones de los actores sociopolíticos. Así, esta corriente se destaca por su particular interés 
en la dimensión temporal de la política. Más específicamente se preocupa por los "legados duraderos" que le dan forma a ciertas prácticas de gobierno (Faletti, 2013).

Para Paul Pierson (2004) la configuración de los legados duraderos pueden ser analizados desde el concepto de "dependencia del rumbo o camino" (path dependence) pensado originalmente para realizar comparaciones, nos permite conceptualizar el desarrollo del gobierno de la educación en Santa Fe. Cómo las instituciones generan en su inercia institucional un efecto de retroalimentación reforzada por el paso del tiempo (Pierson, 2004). Este mismo autor desarrolla el concepto de increasings returns (rendimientos crecientes). Concepto proveniente de la teoría económica, el cual, nos permite pensar en la persistencia de determinadas lógicas de funcionamiento de las instituciones, y la resistencia al cambio de las mismas. En otras palabras se plantea la idea de que hay un rumbo que se refuerza con el paso del tiempo en una política pública o en una institución aun cuando los efectos que generan puedan no ser positivos para la sociedad.

Por último el institucionalismo histórico pondera los contextos fundacionales de ciertas instituciones, los cuales son cruciales para entender su posterior trayectoria de desarrollo. Esto es, las instituciones se desarrollan y adaptan a los cambios pero de manera relativa dado que las mismas están constreñidas por las trayectorias propias de su institucionalidad (Thelen, 1999, 2002).

Dicho rastreo lo realizamos también desde un enfoque jurídico-normativo donde cobra relevancia la organización institucional del sistema educativo y la distribución de competencias y atribuciones, ello nos permite "analizar la estructura de las agencias de gobierno, los órganos intervinientes y las normas que los regulan, estudiándose así los núcleos de control de la educación" (Ruiz, 2013, p. 125).

En este sentido, tomar como referencia un ámbito como el Consejo General de Educación como ámbito estatal donde existía una representación política de actores en la toma de decisiones más amplia que la que sobrevino no quiere decir que la misma haya sido democrática. Pero creemos que la coyuntura actual se presta para debatir acerca de las posibilidades de ampliar los actores que intervienen en los procesos de deliberación y decisión relativas a las políticas educativas y los procedimientos para elegir a dichos actores en la provincia de Santa Fe.

\section{Las Coyunturas Críticas y la trayectoria del desarrollo en el marco de la modernización excluyente}

Antes de sumergirnos en las coyunturas críticas debemos hacer referencia a procesos históricos que configuraron de determinada manera.

Las políticas educativas de los Gobiernos Justicialistas de 1943-1955 fueron acompañadas por los Gobiernos de Suárez (1946-49), Cesar (1949-1952) 
Revista de la Escuela de Ciencias de la Educación, año 13, nRo. 12, vol. 2, julio a diciembre de 2017. Páginas 129147. ISSN 1851-6297. ISSN 2362-3349 (EN LínEA). DEMOCRATIZAR EL GOBIERNo DE LA EDUCACIÓN. HISTORIA RECIENTE Y coyunturas criticas del subsistema educativo en Santa Fe. Mariano Sironi.

y Cárcamo (1952-1955) quienes llevaron adelante cambios en sintonía con los acontecimientos a nivel nacional, es decir, centralización administrativa, expansión de la burocracia y avance del Poder Ejecutivo sobre la instancia legislativa modificando aspectos del gobierno de la Educación en Santa Fe (4).

En este período se sancionó la Ley que técnicamente sigue vigente y que suprimió temporalmente el Consejo General de Educación. Como aclara Ossanna y otros

La reestructuración fundamental se dio en el año 1949 cuando la reforma de la constitución provincial obligó a dictar una nueva ley de educación. La Ley de educación $N^{\circ} 3554$ derogó la del año 1886. Suprimiendo el Consejo General de Educación. En su lugar se estableció que la dirección y administración de la educación estuviera a cargo del Ministerio del ramo, el que contaría con las siguientes Direcciones Generales: de jardines de infantes, primarias, Normales y orientación profesional; de escuelas técnico-profesionales para varones, de escuelas técnico-profesionales para mujeres; y las Direcciones de Educación Física, de Personal y de Administración (Ossanna, et. al. 1997, p. 379).

Para Bacolla (2010) durante este período existió un proceso de centralización que en el aspecto educativo se plasmó en las reformas legales realizadas en continuidad con innovaciones sugeridas desde el nivel nacional. Es decir, una tendencia a la centralización uniformizante consistente en el avance del Estado Nacional en materia de creación y administración de establecimientos, como así también en materia de un fuerte control en las orientaciones curriculares y académicas, como de los docentes.

Con el derrocamiento de Perón en setiembre de 1955 las normas y políticas educativas del período serán revisadas y modificadas en el marco de iniciativas que disolvieron y proscribieron al Partido Peronista (Decreto №3855/55); como así también la difusión de propaganda y/o elementos de afirmación ideológica del Peronismo (Decreto 4161/56). El caso de Santa Fe es analizado por Ascolani (2001) quien analiza la desarticulación de las políticas educativas justicialistas en Santa Fe como así también los procesos de persecución y desplazamiento de docentes y directivos dentro del Sistema educativo por su participación durante el gobierno derrocado.

En este período, luego de la intervención militar (1955-1958), nuestra provincia estará gobernada por dirigentes de origen radical (Silvestre Begnis, 1958-62 y Tessio 1963-1966) que le imprimirán un rasgo de continuidad a las políticas educativas enmarcadas en las perspectivas desarrollistas.

En lo relativo al gobierno de la Educación se restableció el Consejo General de Educación mediante el Decreto Ley № 08199/1956, que sería ratificado por Ley N 4877 en 1958.

El Consejo General estaría integrado por un presidente designado por el Gobernador y acompañado por representantes del Ministerio de Educación, del 
Revista de la Escuela de Ciencias de la Educación, año 13, nRo. 12, vol. 2, julio a diciembre de 2017. Páginas 129147. ISSN 1851-6297. ISSN 2362-3349 (EN LINEA). DEMOCRATIZAR EL GOBIERNO DE LA EDUCACIÓN. HISTORIA RECIENTE Y coyunturas criticas del subsistema educativo en Santa Fe. Mariano Sironi.

Magisterio y de las cooperadoras escolares respectivamente. Es decir se produce una ampliación de los intervinientes en la toma de decisiones de las cuestiones educativas en temas tales como por ejemplo el nombramiento de docentes.

De este modo, se llevó adelante un fundamental reordenamiento normativo que se plasmó en el Digesto escolar sancionado en mayo de 1961 mediante el Decreto № 4720 con el que se reunió el conjunto de normas dispersas que regulaban el nivel primario, constituyéndose en los hechos en un reglamento para dicho nivel.

Otras dos cuestiones relevantes remiten, por un lado a la creación de la Dirección General de Educación Técnica en el ámbito del Ministerio, y a la creación del Estatuto de Establecimientos Privados de enseñanza mediante la Ley 5501/ 61(5).

Este nuevo organismo se creó a partir de una concepción que se sustentaba en el principio del derecho a enseñar y a aprender consagrado en la Constitución Nacional. Defendía la iniciativa privada en materia educativa, basándose en el derecho de los padres a elegir la educación para sus hijos. Para complementar esta medida al año siguiente mediante el Decreto provincial $\mathrm{N}^{\circ}$ $5411 / 62$ se plantea la necesidad de crear un cuerpo de inspectores para la Educación Privada. Este organismo antecede la creación del subsistema de educación privada de 1968 como veremos más adelante.

Vale agregar que en este período se realizó una reforma constitucional que sigue rigiendo para la provincia. En su texto, contiene un capítulo específico para la educación en donde se menciona explícitamente los principios de defensa y protección del derecho a la libertad de enseñanza y a la elección de los padres sobre los establecimientos educativos. Sin embargo, a la par el rol del Estado es ratificado. Nada se plantea en estos artículos sobre el Gobierno de la Educación. Las disposiciones relativas a las funciones de los ministros y la decisión del presupuesto para educación y/o adhesión a leyes educativas nacionales se enmarcan en lo dispuesto por las atribuciones del Poder Ejecutivo (Art. 72) y la legislatura (Art. 55) (6).

Es decir, la estructura normativa e institucional que configuraron los gobiernos desarrollistas en Santa Fe, retrotrajo cierta institucionalidad previa a los gobiernos Justicialistas a la par que introdujo innovaciones propias de la visión predominante. A partir del golpe del 66, la autodenominada Revolución Argentina, llevó adelante su proyecto de Modernización excluyente que con el objetivo de limitar los elementos democratizadores del sistema educativo comenzó a neutralizar el rol del Estado Nacional en materia educativa "...y fueron tomando cada vez más peso otros dos agentes, los estados provinciales por un lado, y el sector privado por el otro" (Pineau, 2006, p. 30).

Como veremos a continuación esta tendencia se profundizará a partir de la autodenominada Revolución Argentina, dictadura que gobernó el país entre 1966-1973. Es en este período donde ubicamos la primera coyuntura crítica en la que se gesta la institucionalidad del gobierno de la educación en Santa Fe. 
Revista de la Escuela de Ciencias de la Educación, año 13, nRo. 12, vol. 2, julio a diciembre de 2017. Páginas 129147. ISSN 1851-6297. ISSN 2362-3349 (EN LínEA). DEMOCRATIZAR EL GOBIERNo DE LA EDUCACIÓN. HISTORIA RECIENTE Y coyunturas criticas del subsistema educativo en Santa Fe. Mariano Sironi.

\section{Primer Coyuntura crítica: la centralización y la creación del Servicio Pro- vincial de Enseñanza Privada}

El Estado Burocrático Autoritario (O’Donnell, 1975) que comienza a configurarse con la dictadura militar autodenominada Revolución Argentina, implicó para nuestra provincia una marcada dependencia y subordinación de la orientación de la política educativa nacional (7).

A nivel nacional y provincial, la conducción educativa provincial, en general, quedó en manos de sectores ligados al catolicismo que promovió la creciente autonomización del sector privado de la educación. Durante este período se comienza a plasmar fuertemente la retracción del Estado en materia educativa con la derogación de la Ley Láinez (1970) y el crecimiento del subsidio a la educación privada.

En nuestra provincia dos normas de este período son relevantes para nuestro análisis. En primer lugar la Ley de Facto 6247/68 cuyo decreto reglamentario 2880/69 habilitó el desarrollo de una estructura estatal interna bajo el control directo de los sectores de la Educación Privada, el Servicio Provincial de Educación Privada (SPEP). Como sostiene Puebla

De acuerdo a la estructura de gobierno que se le asigna al SPEP dispone de dependencias que cumplen funciones similares al sector oficial y que no comparten con el sector oficial las instancias de gobierno general, a excepción de la máxima autoridad de la cartera el Ministro de Educación. Es decir que el SPEP tiene a su cargo la conducción de cada nivel, la supervisión, las dependencias administrativas, pedagógicas y financieras, con independencia de similares instancias en el sector oficial, situación que afirma el paralelismo anteriormente planteado (Puebla, 2009, p. 63).

Esta situación convivirá, a su vez, con la fuerte incidencia de la Iglesia católica en la orientación de la política educativa de la provincia en el sistema de educación pública y la posibilidad de igualar la escuela pública y la privada desde una perspectiva en la que se pondera el rol subsidiario del Estado (8).

Otra consecuencia de la política educativa de esta dictadura es la tendencia a la centralización de las decisiones en el ejecutivo provincial a través del Ministerio de Educación que a partir de decretos, resoluciones y circulares tenderá a concentrar la toma de decisiones en el ejecutivo y, paralelamente garantizará amplia autonomía al sector privado permitiendo que el mismo funcione prácticamente como un subsistema, situación que como veremos persistirá en democracia (Veleda y Cardini, 2002).

Esta derivación es producto de la segunda norma que queremos destacar, la ley de facto $N^{\circ} 6553 / 69$ que reforma la estructura de ministerios en 1969 y habilita la anulación del Consejo General de Educación.

Es decir, en este período se consolidan dentro del subsistema de educación provincial dos estructuras institucionales paralelas dentro del mismo 
Ministerio. Este es el principal legado de esta coyuntura en términos de institucionalidad del gobierno de la educación en Santa Fe.

Junto con la situación de fortalecimiento del actor mencionado, ya que por un lado tiene un mayor peso específico en el universo de instituciones educativas privadas, logrando ubicar en los cargos de decisión política a dirigentes ligados a la misma. Por otro lado, en estos tiempos de dictadura su influencia ideológica y el aporte de cuadros de gestión también es relevante en el sistema educativo público. Esta situación es acorde con el proceso que se venía consolidando a nivel nacional.

\section{Segunda Coyuntura crítica: el desmantelamiento definitivo del Consejo General de Educación y la consolidación de la trayectoria institucional en democracia}

Con el golpe de Estado del 24 de marzo de 1976 se consolida la represión ya esbozada a partir de la asunción de Oscar Ivanissevich como Ministro de Educación en 1974 durante el gobierno de Isabel M. de Perón.

Si bien existen discusiones alrededor del grado de homogeneidad de las políticas educativas del Proceso debido a la discontinuidad de las gestiones mas allá de que la misma estuvo caracterizada por la fuerte presencia de funcionarios ligados a las Fuerzas Armadas y al Consejo Superior de Educación Católica (Rodríguez, 2011), creemos con Kaufmann (2006) en la existencia de algunas líneas directrices de la política tales como

(...) matriz ideológica subyacente (discurso nacionalista integrista, clericalismo, visión provindecialista y conservadurismo intelectual) (...) los rasgos distintivos que las signaron (...) se destacan: el desarme del Estado docente y la progresiva derivación de las responsabilidades educativas al ámbito privado; la militarización educativa; el dispositivo pedagógico procesista; el disciplinamiento docente/estudiantil; las cesantías; persecuciones; los microprocesos de violencia institucional centrados en las censuras y depuraciones bibliográficas; las proscripciones en la organización gremial; (...) la impregnación de espíritu confesional e ideas creacionistas en el campo educativo (...); por citar los componentes más representativos (Kaufmann, 2006, p. 151).

Quien conduciría estas líneas de acción en Santa Fe sería el Capitán de Navío (retirado) Orlando Pérez Cobo cuyo primer acto de gobierno fue intervenir el Consejo General de Educación mediante un decreto ( $\mathrm{N}^{\circ} 0065 / 1976$ ) desplazando de sus cargos a las autoridades del Organismo, cuyas autoridades eran el Profesor Mauricio Epelbaum, (presidente, luego detenido en calidad de preso a disposición del Poder Ejecutivo Nacional) y a los representantes del Ministerio de Educación, de los docentes y de las cooperadoras. El objetivo de esta intervención fue completar el desmantelamiento del Consejo General de Educación iniciado en la dictadura de Onganía. 
Revista de la Escuela de Ciencias de la Educación, año 13, nRo. 12, vol. 2, julio a diciembre de 2017. Páginas 129147. ISSN 1851-6297. ISSN 2362-3349 (EN LínEA). DEMOCRATIZAR EL GOBIERNo DE LA EDUCACIÓN. HISTORIA RECIENTE Y coyunturas criticas del subsistema educativo en Santa Fe. Mariano Sironi.

Esta iniciativa fue complementada con la sanción de dos decretos fundamentales que consolidaron la situación anterior gestada durante el gobierno de la Revolución Argentina, el № 2439 de julio de 1977 y el № 4176 de noviembre de 1977.

El primer decreto cita la norma 6553/68 y ratifica el propósito de reordenar políticas y fines del gobierno provincial mediante una eficiente organización de su administración pública y para ello plantea la necesidad de adecuar las estructuras orgánicas en el ámbito del Poder ejecutivo. Por ello en la resolución se plantea reordenar el Ministerio de educación y cultura con una organización básica hasta departamento.

Este decreto eliminó definitivamente el Consejo General de Educación dado que debajo del ministro establece dos subsecretarías (de cultura y educación) y una secretaría privada y dispone la organización de 12 direcciones (9) con específicas funciones y un Servicio Provincial de educación superior media y técnica.

El segundo decreto, $\mathrm{N}^{\circ} 4176 / 77$ es el que habilita el traslado y/o reasignación de todo el personal del Consejo General de Educación, en realidad esta disposición es una profundización de una política de cesantías y exoneraciones docentes que comenzó en 1975 a nivel nacional a partir del endurecimiento del rol represivo del Gobierno de Isabel Perón (Alonso, 2009, pp. 35-53).

De este modo, con ambas disposiciones quedó eliminado de manera definitiva el cual desde ese momento no se volvió a restablecer.

Paralelamente, se fortaleció aún más el rol del ejecutivo provincial que comienza a ejercer el gobierno de la educación mediante dispositivos legales tales como Decretos, Resoluciones y circulares sin la participación de otros organismos o poderes del estado provincial. Este es el mecanismo legado posteriormente en democracia.

En relación con el Servicio Provincial de Educación Privada (SPEP) durante este gobierno se ampliaron sus atribuciones y funciones y su estructura mediante las leyes Nro. 6978/73, 8381/79. Esto consolidó un subsistema de educación privada con amplia autonomía (Puebla, 2009). Hoy este ámbito sigue funcionando bajo la denominación de Servicio Provincial de Educación Pública de Gestión Privada.

En este sentido, Veleda y Cardini (2002) destacan que el principal beneficiario de esta situación de centralización de las decisiones en el ejecutivo y autonomía del sector privado es la Iglesia católica dada la flexibilidad para crear establecimientos que se le otorgó y el principio de subsidiariedad vigente en los discursos y en los fundamentos de los decretos y normas del período en los cuales la tendencia es a equiparar la educación pública y la privada. De este modo, siendo la Iglesia el principal agente educativo dentro del ámbito privado con capacidad, era además una fuerte influencia en la orientación de la política educativa provincial, replicando en este sentido lo que acontecía a 
nivel nacional. Distintos hechos nos permiten dar cuenta de esta situación que reseñamos brevemente.

En primer lugar, desde 1983 en adelante los dispositivos legales sancionados durante la dictadura fueron ratificados por acción y/u omisión en democracia, por ejemplo las funciones del SPEP se "legalizaron" en democracia con la Ley No 10.878/92.

El gobierno de la educación no se modificó sustancialmente ya que si bien un decreto del año 1986 ( $\left.\mathrm{N}^{\circ} 456\right)$ plantea una desconcentración de carácter administrativo y pedagógico y a inicios de los '90 se produce la regionalización del sistema de educación pública, (primero en 7 regiones y luego en 9 regiones) mediante el decreto 3667/94; las decisiones de fondo siguieron dependiendo exclusivamente de los ministros del área. A partir del año 2007 a esta división del territorio se le yuxtapondrá una nueva regionalización por nodos (10).

Otro dato que va en este sentido lo representa la sanción de la ley $\mathrm{N}^{\circ}$ 10.416 para crear Consejos escolares en 1989, la cual no pudo ser implementada de manera sistemática en el territorio provincial. Es decir, esta norma que promueve una mayor participación no pudo alterar los modos establecidos de decisión al interior de las Escuelas.

En el año 2007, la Ministra de educación del Gobernador Jorge Obeid (2003-2007), la Prof. Adriana Cantero intentó de manera infructuosa promover un debate y posicionamiento en agenda de una Ley Provincial de Educación en el marco de la sanción de la Ley de Educación Nacional № 26.206. Dos cuestiones a tener en cuenta, por un lado, la escasa repercusión que tuvo esta iniciativa a pesar del esfuerzo ministerial de hacer circular un documento en las escuelas con el objetivo de consultar a docentes y directivos de escuelas y convocar a una Mesa de Concertación con Universidades, Cámaras empresariales y Sindicatos. Y por el otro la ausencia en el documento del Consejo General de Educación (Diario EL Litoral, 17 de agosto de 2008, Rosario/12, 14 de octubre, 2007).

En cuanto al Consejo General de Educación, en el año 2010 ingresó a la Legislatura provincial un proyecto de restablecimiento que perdería estado parlamentario (10). Tres años más tarde se presentó un proyecto de creación de un Consejo social de Planeamiento y Evaluación de la Educación (11) con funciones y atribuciones del anterior organismo. Este proyecto al igual que el anterior cayó en el olvido.

\section{¿Consolidación del subsistema de gobierno de la educación en democracia? Conclusiones provisorias}

En el presente trabajo presentamos una hipótesis: las coyunturas críticas de 1966-1973 y 1976-1983, generaron una institucionalidad y modos de gobernar la educación que se consolidaron dando cuenta de un caso de de- 
Revista de la Escuela de Ciencias de la Educación, año 13, nRo. 12, vol. 2, julio a diciembre de 2017. Páginas 129147. ISSN 1851-6297. ISSN 2362-3349 (EN LínEA). DEMOCRATIZAR EL GOBIERNo dE LA EDUCACIÓN. HISTORIA RECIENTE Y coyunturas criticas del subsistema educativo en Santa Fe. Mariano Sironi.

pendencia del rumbo. En este sentido, en la actualidad esta institucionalidad constituye una fuerte dificultad a la que se enfrentarán los proyectos de Leyes Provinciales de Educación presentados.

En el desarrollo intentamos presentar el proceso de supresión del Consejo General de Educación y el surgimiento de determinados núcleos institucionales desde los que se ejerce el control y gobierno de la educación de manera centralizada a través de Decretos, Resoluciones y Circulares por parte del Ministro de Educación y sus funcionarios. Es decir, se consolidó un legado institucional en el gobierno de la educación cuya característica es la ausencia de ámbitos formales de deliberación y consulta con los actores del sistema educativo por parte del Ejecutivo de turno.

Así en nuestro recorrido encontramos que el Poder Ejecutivo provincial a través del Ministerio de Educación, y dentro del mismo el SPEP, son los ámbitos en los cuales se gobierna de manera centralizada la política educativa en la jurisdicción a partir de ratificar normas precedentes y mecanismos institucionales preexistentes.

Estos ámbitos y su institucionalidad fueron configurándose en tiempos de dictadura y adquirieron cierta inercia consolidada en democracia durante los gobiernos del período 1983-2007. Por ello, cuando hablamos de la consolidación de un subsistema de gobierno con instancias formales e informales, nos referimos a que esta recentralización del gobierno de la educación a partir de las reformas de los 90 a la que se hace referencia en la literatura, en realidad comenzó a gestarse en coyunturas críticas anteriores a las reformas y creemos que tendría una dependencia del desarrollo en el que los cambios de estas políticas reforzaron la misma.

Sin embargo, en los últimos años ciertas medidas como la creación de Ruedas de Convivencia, la Ley de Centros de Estudiantes N ${ }^{\circ} 13.392$, y la Ley Provincial № 12.967 de "Promoción y protección integral de los derechos de las niñas, niños y adolescentes" cuyos arts. № 14 y 15 contienen un concepto de derecho a la educación en tanto derecho humano fundamental.

De este modo consideramos que revisar de manera crítica la institucionalidad del Gobierno de la Educación puede redundar en pensar un proyecto de Ley Provincial de Educación que habilite y potencie espacios de deliberación que forme ciudadanos con capacidad de intervenir en la conformación de redes dialógicas horizontales con capacidad de interpelar y cuestionar al poder. Esto es promover una ley que instituya ámbitos de deliberación y decisión para los actores que están implicados en las políticas educativas lo cual podría redundar en un fortalecimiento de la ciudadanía en relación al ejercicio de sus derechos, es decir a la democratización.

El restablecimiento de un Consejo General de Educación, Consejos Regionales y Consejos Escolares con atribuciones definidas podría ser un hecho positivo para fortalecer la exigibilidad del derecho a la educación como 
Revista de la Escuela de Ciencias de la Educación, año 13, nRo. 12, vol. 2, julio a diciembre de 2017. PÁginas 129147. ISSN 1851-6297. ISSN 2362-3349 (EN LINEA). DEMOCRATIZAR EL GOBIERNO dE LA EDUCACIÓN. HISTORIA RECIENTE Y coyunturas criticas del subsistema educativo en Santa Fe. Mariano Sironi.

derecho fundamental que habilita y fortalece el ejercicio de otros derechos ciudadanos. Es sabido que este tipo de espacios, en la medida en que estén conformados de manera representativa pueden potenciar la valoración de las decisiones colectivas dentro de las democracias (Urfalino, 2013). A su vez, la posibilidad de ampliar los criterios para habilitar mas actores en los mecanismos de producción de políticas educativas en una Ley Provincial de Educación, no solo es una decisión estratégica en el largo plazo respecto a la forma de gobierno (las instituciones formales del subsistema de gobierno educativo) y al modo de gobernar los conflictos inherentes a la vida en sociedad, sino también una forma de promover una cultura política que asuma que la deliberación cuanto más representativa y participativa será más democrática en las formas de resolver la conflictividad inherente a la vida en sociedad.

\section{Notas bibliográficas}

(1) La provincia de Santa Fe está ubicada en la región centro-este de la República Argentina, al sur del Continente Americano. La superficie total que ocupa es de $133.007 \mathrm{~km}^{2}$. En su eje norte-sur, tiene una longitud de $720 \mathrm{~km}$ y en su eje esteoeste alcanza los $380 \mathrm{~km}$. Está dividida políticamente en 19 departamentos y su ciudad capital es Santa Fe de la Vera Cruz. Esta ciudad está ubicada a 475 km de la Capital Federal, provincia de Buenos Aires (Ver: www.santafe.gob.ar). Su sistema educativo es el cuarto más grande del país: 4816 establecimientos educativos, 835.250 alumnos/as, y más de 55 mil docentes (Anuario estadístico 2015). Para un abordaje de la historia de Santa Fe se recomienda la lectura de la "Nueva Historia de Santa Fe" (12 tomos). Editada conjuntamente por Prohistoria Eds. y Diario de Editorial La Capital.

(2) En marzo del presente año el Diputado Provincial Alejandro Boscarol (UCR-Frente Progresista Cívico y Social-FPCyS) presentó un proyecto de Ley acompañado en la presentación por los Diputados provinciales de la UCR. En Abril el Diputado Provincial Rubén Giustiniani (PS-FPCyS) presentó otro proyecto acompañado por los Diputados Provinciales Silvia Augsburger, Mercedes Meier y Carlos Del Frade. Finalmente en agosto-setiembre del presente año el Diputado Provincial Carlos Del Frade (FSP) y la Diputada Provincial Verónica Benas (Partido PARESFPCyS) presentaron sendos proyectos. Asimismo el Gobernador Miguel Lifschitz del FPCyS planteó en el discurso inaugural de las sesiones legislativas del $1^{\circ}$ de mayo la necesidad de sancionar una ley de educación provincial (Diario La Capital y Diario el Litoral, 2 de mayo de 2016). A partir de este planteo en Julio del presente año la Ministra de Educación Claudia Balagué inició a partir de fines de Julio una ronda de consultas por toda la provincia y a través de Internet para llegar a fin de año con un "documento síntesis a partir del cual construir un proyecto de Ley Provincial de Educación" (Diario El Litoral y Diario La Capital 6 de julio de 2016). En el caso del Proyecto de Benas no se propone el restablecimiento del Consejo Provincial de Educación, aunque sí propone efectivizar la organización de los Consejos Escolares establecidos en la Ley № 10.416 del año 1989. 
Revista de la Escuela de Ciencias de la Educación, año 13, nRo. 12, vol. 2, julio a diciembre de 2017. Páginas 129147. ISSN 1851-6297. ISSN 2362-3349 (EN LínEA). DEMOCRATIZAR EL GOBIERNo dE LA EDUCACIÓN. HISTORIA RECIENTE Y coyunturas criticas del subsistema educativo en Santa Fe. Mariano Sironi.

(3) Para un desarrollo de los temas y problemas de la corriente neo-institucionalista ver Peters, G. (2003) El nuevo institucionalismo.

(4) La Ley de Educación Común de 1886 sancionada durante la gobernación de José Galvez (1886-1890) contenía la mirada innovadora de la época propia de la Ley $N^{\circ} 1420$ en cuanto al gobierno y administración del sistema educativo y la permanencia de la enseñanza de la religión católica en el currículum de las escuelas provinciales. Respecto del Gobierno de la Educación, el Consejo General de Educación fue investido de determinadas facultades de manera ambigua; ya que si bien podía intervenir en la elaboración del presupuesto, administrar los fondos y bienes escolares, intervenir en la designación y cesantía del personal docente, sus decisiones quedaban supeditadas a la expresión de conformidad del Ministro de Instrucción pública (Ossanna, 1997).

(5) "El mismo fijaba dos categorías de establecimientos; los autorizados, con capacidad para expedir títulos, y los incorporados, que percibían el aporte estatal para funcionar. En ambos casos las remuneraciones del personal escolar se equipararían a los sueldos del personal similar de las escuelas oficiales. Como órgano de gobierno y administración se crearía el Consejo General de Establecimientos Privados de Enseñanza, integrado por dos representantes del Ministerio, uno de los establecimientos privados, uno de los padres de los alumnos y uno de los docentes designado por sufragio universal, secreto y obligatorio. La fiscalización técnica y administrativa quedaría a cargo del Estado" (Osanna y otros 1993, p. 408).

(6) La Constitución de la provincia de Santa Fe fue reformada en pleno proceso de destitución de Frondizi (1962) e intervención federal de todas las provincias que anularon los procesos electorales que eligieron las autoridades provinciales que hubieran debido gobernar a partir de ese año. Luego mediante un decreto $\left(N^{\circ} 4430 / 62\right)$ se dejó sin efecto dicha nulidad para aquellos que fueron elegidos como constituyentes, en consecuencia la Constitución Provincial reformada de 1962 siguió en vigencia hasta el golpe de 1966. Con el nuevo proceso electoral convocado en 1963, la provincia será gobernada por el Dr. Aldo Tessio de la UCRP, y como decíamos más arriba, lo que se destacan son los rasgos de continuidad en materia de política educativa.

(7) En este período el Ministerio de Educación se reconvirtió en Secretaría de Estado bajo dependencia del Ministerio del Interior. Braslavsky (1985) señala que en este gobierno hay un planteo de reforma integral del sistema educativo (Proyecto de Ley Orgánica) que no llegan a cumplirse, aunque se constituyen en los antecedentes de las reformas de los años siguientes. Asimismo en estos años se creó el Consejo Federal de Educación (1972) que reemplazó al centralizado Consejo Nacional de Educación.

(8) Ejemplifica la concepción el planteo del ex - Ministro de estos gobiernos: "El sistema educativo provincial se nutre en dos vertientes, la escuela pública oficial y la pública privada. Ambas se sustentan en el primer núcleo educador: LA FAMILIA, a la cual reconocemos, por derecho natural y primigenio, la capacidad de opción que nuestra acción de gobierno debe auspiciar, cumpliendo en éste como en otros 
Revista de la Escuela de Ciencias de la Educación, año 13, nRo. 12, vol. 2, julio a diciembre de 2017. Páginas 129147. ISSN 1851-6297. ISSN 2362-3349 (EN LINEA). DEMOCRATIZAR EL GOBIERNO dE LA EDUCACIÓN. HISTORIA RECIENTE Y

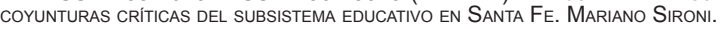

campos (...) el principio de SUBSIDIARIEDAD” (Ex Ministro Sutter Schneider, citado en Ossanna et. al. 1997, p. 418). La otra cuestión relacionada con el desarrollo del SPEP es el crecimiento del sector privado en los niveles medio y superior no universitario, especialmente establecimientos terciarios orientados a la formación docente.

(9) Fabiana Alonso (2009) en un interesante trabajo sobre la educación pública durante el Proceso de Reorganización Nacional en Santa Fe, plantea que mediante este decreto se crea la Dirección Provincial de Educación Privada, sin embargo en las fuentes digitalizadas del Archivo de Decretos de la Provincia de Santa Fe, el mencionado decreto no presenta ninguna mención a dicha Dirección. Alonso F. (2009) “El combate ideológico en la educación pública satafesina: 1976-1983”. Ed. UNL: Santa Fe.

(10) "La regionalización se plantea como una estrategia de organización y gestión del territorio, con la finalidad de disminuir desequilibrios socio-económicos y acercar el Estado fortaleciendo su proximidad". De este modo se propuso dividir de la siguiente manera: Región 1 - Nodo Reconquista; Región 2 - Nodo Rafaela; Región 3 - Nodo Santa Fe; Región 4 - Nodo Rosario; y Región 5 - Nodo Venado Tuerto. Ver en Plan Estratégico provincial de Santa Fe.https://www.santafe.gov.ar/index. php/plan_estrategico_provincial/content/view/full/110705.

(11) Presentado por Hugo Marcucci del Frente Progresista Cívico y Social el 2 de noviembre de 2010.

(12) Presentado por el Diputado Provincial y ex Rector de la Universidad Nacional de Rosario Juan Carlos Millet el 29 de agosto de 2013.

\section{Referencias bibliográficas}

- Abratte, J. P. (2010). Las reformas educativas de los 80 y los 90 en la Provincia de Córdoba, entre el imaginario democrático y el neoliberal. En Roitenburd, S. y Abratte, J. P. (Comps.) Historia de la Educación en Argentina. Del discurso fundante a los imaginarios reformistas contemporáneos. Córdoba: Editorial Brujas.

- Acuña, C. y Chudnovsky, M. (2007). La gobernabilidad de los sistemas educativos: Una metodología para su análisis y mejoramiento. Seminario Internacional Gobernabilidad de los Sistemas Educativos en América Latina organizado por el Centro de Estudios de las Políticas Públicas (CEPP) y el Ministerio de Educación, Cultura y Tecnología de la República Argentina. 18 y 19 de octubre de 2007

- Alonso, F. (2009). El combate ideológico en la educación pública santafesina: 19761983. Santa Fe: Editorial UNL.

- Ascolani, A. (2001). La fuerza: ¿Derecho de las bestias o de la razón?, Ciudadanía restringida y Educación en Argentina (1955-1958)". En Anuario de la Sociedad Argentina de Historia de la Educación, №3, pp. 113-137

- Bacolla, N. (2014). Política, administración y gestión en el peronismo santafesino, 1946-1955. En Macor, D. y Tcach, C. (editores) La invención del peronismo en el interior del país.2da edición. Santa Fe: Ediciones UNL.

- Braslavsky, C. (1985). La educación argentina. 1955-1980. El país de los argentinos. Buenos Aires: Centro Editor de América Latina. 
Revista de la Escuela de Ciencias de la Educación, año 13, nRo. 12, vol. 2, julio a diciembre de 2017. Páginas 129147. ISSN 1851-6297. ISSN 2362-3349 (EN LínEA). DEMOCRATIZAR EL GOBIERNo dE LA EDUCACIÓN. HISTORIA RECIENTE Y coyunturas criticas del subsistema educativo en Santa Fe. Mariano Sironi.

- Carletti, G. (2005). La descentralización educativa en Argentina: un análisis comparativo de la gestión escolar. I Congreso Nacional de Estudios Comparados en Educación "Retos para la Democratización de la Educación. Perspectiva Comparada", Buenos Aires, 18 y 19 de noviembre.

- DINIECE. (2015). Anuario Estadístico Educativo 2015. Dirección Nacional de Información y Estadística Educativa. En: http://portales.educacion.gov.ar/ diniece/2016/09/20/anuario-estadistico-educativo-2015/

- Falleti, T. (2004). Descentralización educativa en argentina: condicionantes institucionales y Consecuencias. En e-l@tina. Revista electrónica de estudios latinoamericanos, Vol. 2, nº 8, Buenos Aires, julio-setiembre de 2004, pp. 17-36. En http://www.catedras.fsoc.uba.ar/udishal

- - ----; Sheingate A. (2013). "Historical Institutionalism in Political Science". En The Oxford Handbook of Historical Institutionalism. Ed. Orfeo Fioretos.

- Feldfeber, M. (Comp.) (2009). Autonomía y Gobierno De La Educación. Perspectivas, Antinomias y Tensiones. Buenos Aires: Editorial Aique.

- Filmus, D. (1997). La descentralización educativa en argentina: elementos para análisis de un proceso abierto. Ponencia presentada en el Coloquio Regional sobre Descentralización de la Educación en América Central, Cuba y República Dominicana (1997 Nov. 3-5: San José). CLAD; Países Bajos. Gobierno Nacional; Costa Rica. Ministerio de Planificación Nacional y Política.

- Giovine, R. (2012). El arte de gobernar al sistema educativo: discursos de estado y redes de integración socioeducativas. Colección Cuadernos Universitarios. Bernal: Universidad Nacional de Quilmes Editorial.

- Gorostiaga, J. J. y Vieira, L. F. (2011). Tendencias nacionales y sub-nacionales en el gobierno escolar: Argentina y Brasil 1990-2010. En D.A. Oliveira, M. Pini y M. Feldfeber (orgs.), Políticas educacionais e trabalho docente: perspectiva comparada, Argvmentvm Editora / Fino Traço Editora. En prensa.

- Grinberg, S. (2008). Educación y poder en el sigloXXI. Gubernamentalidad y Pedagogía en las sociedades de gerenciamiento. Buenos Aires: Miño y Dávila Editores.

- Kaufmann, C. (Dir.) (2006). Dictadura y Educación. Los textos escolares en la historia argentina reciente. Tomo I. Buenos Aires: Miño y Dávila.

- Gonzalez, L. y Lardone, M. (2013). Federalismo y política subnacional en perspectiva comparada: algunos desarrollos, interrogantes y potenciales agendas de investigación. En Falleti T; González, L. y Lardone, M. El federalismo argentino en perspectiva comparada. Córdoba: EDUCC.

- Nosiglia M. y Rebello, G. (2005). La concertación educativa. Una aproximación al estilo de definición e implementación de las políticas de Transformación educativa. En Argentina de los 90. En Revista del IICE. p. 3 - 18.Ed. Miño y Dávila y UBA

- O’Donnell G. (2010). Democracia, agencia y estado. Teoría con intención comparativa. Buenso Aires: Ed. Prometeo libros.

$\bullet$ (1982). El Estado Burocrático Autoritario. Buenos Aires: Ed. Belgrano.

- Ossanna, E;. Ascolani, A; Moscatelli, M. y Pérez A. (1997). "Una aproximación a la educación santafesina de 1885- 1945". En Puiggrós A. (Dir.) La Educación en las 
Revista de la Escuela de Ciencias de la Educación, año 13, nRo. 12, vol. 2, julio a diciembre de 2017. PÁginas 129147. ISSN 1851-6297. ISSN 2362-3349 (EN LINEA). DEMOCRATIZAR EL GOBIERNO dE LA EDUCACIÓN. HISTORIA RECIENTE Y coyunturas criticas del subsistema educativo en Santa Fe. Mariano Sironi.

Provincias y Territorios Nacionales (1885-1945). Tomo IV. Buenos Aires: Editorial Galerna.

1983”. En Ossanna E. (Coord.) La educación en las provincias y territorios nacionales. Buenos Aires: Editorial Galerna.

- Paviglianiti, N. (1996). Aproximaciones al desarrollo histórico de la Política Educacional. Revista Praxis Educativa, UNPAM, Año II N² 2. (pp. 3-8).

- Pierson, P. (2004). Politics in time. History, institutions, and social analysis. New Jersey: Princeton University Press.

- Pineau, P. (2006). Impactos de un asueto educacional. Las políticas educativas de la dictadura (1976-1983). En Pineau, P., Mariño, M., Arata, N. y Mercado, B. (eds.) El principio del fin. Políticas y memorias de la educación en la última dictadura militar (1976-1983). Buenos Aires: Colihue. p.13-111.

- Plomé, A. (2002). Reforma del estado y descentralización educativa. El caso de la provincia de Santa Fe (1992-1995). Rosario: UNR Editora.

- Pronko, M., Vior, S. (1999). Consejo Federal de Cultura y Educación ¿espacio para la coordinación interjurisdiccional o para la legitimación de decisiones centralizadas? En Vior, S. (Dir.) Estado y educación en las provincias. Buenos Aires: Ed. Miño y Dávila.

- Puebla, M. (2009). La legislación del subsistema privado: el caso de la provincia de Santa Fe. En Políticas Públicas, Campinas, v.2, n.2, p.54-70, dez. 2009 - ISSN: 19823207.

- Rivas, A. (2004). Gobernar la educación. Estudio comparado sobre el poder y la educación en las provincias. Buenos Aires: Gránica.

- Rodriguez, L. (2011). Católicos, nacionalistas y políticas educativas en la última dictadura (1976-1983). Rosario: Prehistoria Ediciones.

- Ruiz, G. (2013). El Enfoque Jurídico normativo de la política educacional. En Tello, C. (coord. y comp.) Epistemologías de la Política educativa, Posicionamientos, perspectivas y enfoques (pp. 109-161). Sao Paulo: Campinas: Ed. Mercado de Letras.

- Santa Fe: Ubicación, Datos estadísticos y símbolos En https://www.santafe.gov.ar/ index.php/web/content/view/full/93661

- Thelen, K. (1999). Historical institutionalism in comparative politics. Annual Review of political science, 2. 384-401

- Thelen, K. (2002). The explanatory power of Historical institutionalism. En Mayntz. (Ed. Akteure -Mechanismen -Modelle. Zur Theoriefähigkeit makro-sozialer Analysen. 2002 pp. $91-108$.

- Urfalino, P. (2013). Cerrar la Deliberación. Teoría de la decisión Colectiva. Buenos Aires: Prometeo.

- Veleda, C. y Cardini, A. (2002). Provincia de Santa Fe. En Rivas A. Proyecto: Las Provincias Educativas. Estudio comparado sobre el Estado el Poder y la educación en las 24 Jurisdicciones. Informe $\mathrm{N}^{\circ} 2$. Disponible en: http://www.cippec.org/ documents/10179/58548/Serie+informes+provinciales+-+Santa+Fe.pdf/4b01bd173fc5-41e9-8d36-56b9dd0e4ca3 
Revista de la Escuela de Ciencias de la Educación, año 13, nRo. 12, vol. 2, julio a diciembre de 2017. Páginas 129147. ISSN 1851-6297. ISSN 2362-3349 (EN LíNEA). DEMOCRATIZAR EL GOBIERNO DE LA EDUCACIÓN. HISTORIA RECIENTE Y coyunturas criticas del subsistema educativo en Santa Fe. Mariano Sironi.

\section{Documentos y Leyes}

- Constitución Nacional Argentina

- Constitución de la Provincia de Santa Fe

- Ley de Educación Provincial No 3554/49

- Ley Provincial $\mathrm{N}^{\circ} 2369 / 32$

- Decreto $\mathrm{N}^{\circ} 4720 / 61$

- Decreto $N^{\circ}$ 0065/1976 (Gobierno de facto)

- Ley provincial N 6247/68 (Gobierno de facto)

- Plan Estratégico de la Provincia de Santa Fe

- $\quad$ Decreto- Ley provincial $N^{\circ} 6553 / 68$

- Ley Provincial № 12.967 de "Promoción y protección integral de los derechos de las niñas, niños y adolescentes"

- Ley Provincial N 13392. Sobre Centros de Estudiantes

- Diarios Consultados (versiones on-line)

- La Capital - Rosario. www.lacapital.com.ar

- El Litoral Santa Fe www.ellitoral.com.ar

- Rosario 12 www.pagina12.com.ar/rosario12

- EL Litoral - Santa Fe 